MATEC Web of Conferences 37, 01007 (2015)

DOI: $10.1051 /$ matecconf $/ 20153701007$

(C) Owned by the authors, published by EDP Sciences, 2015

\title{
NUMERICAL ANALYSIS OF FLUID PARTICLES MOTION IN CURVED DUCTS
}

\author{
L.A. Belyaev ${ }^{1}$, A.S. Zaitsev ${ }^{1}$, A.A. Kondakov ${ }^{1,}$ a, S.A. Shevelev ${ }^{1}$, E.P. Valkov ${ }^{1}$, A.A. Matveeva ${ }^{1}$ \\ National Research Tomsk Polytechnic University, Tomsk, Russia
}

\begin{abstract}
The developed mathematical model allows determining the fields of speeds and pressure, when viscous incompressible fluid flows in 2-dimensional variable cross-section ducts at laminar flow mode. The dynamics of the condensed moisture is determined by the differential equation of the motion amount for the particle. Solving the equation the trajectories of the moisture drops motion and its speed in continuous medium flow.
\end{abstract}

\section{Introduction}

In turbines of atomic power stations (APS) the major part of the stages operates in the region below the saturation curve. Economic efficiency and erosion wear of the stages depend significantly on the moisture degree of the steam incoming to blades. Increase of the average steam moisture by $1 \%$ results in decrease of internal relative efficiency of the turbine by $1 \%$ [1]. One of the most effective ways is moisture entrainment by means of different separating devices. In the majority of APS turbines steam separation is used after the high-pressure cylinder in inertia-type separators.

The purpose of the work is numerical study of moisture drops motion and deposition in complicated passages typical for separation devices paths.

\section{Mathematical model and study technique}

The basis of the mathematical model for the flux flow in the duct is presented by Navier-Stokes equations. The algorism SIMPLE by Patankar-Spolding was used as the solution method [2, 5].

Digitization of initial equations was performed using the control volume (CV) approach. Due to reasons mentioned in [2], orthographical staggered grid will be used.

Let us consider the problem of stationary laminar flow of viscous incompressible liquid in 2-dimensional straight duct.

Liquid with the density $\rho$ and dynamic viscosity $\mu$ flows into 2-dimensional duct with the length $L$ and height $H$.

Mathematical model of the problem under consideration looks as follows.

${ }^{a}$ Corresponding author : kondakov_alexandr@tpu.ru 


$$
\left\{\begin{array}{l}
\frac{\partial(\rho u u)}{\partial x}+\frac{\partial(\rho v u)}{\partial y}=\frac{\partial}{\partial x}\left(\mu \frac{\partial u}{\partial x}\right)+\frac{\partial}{\partial y}\left(\mu \frac{\partial u}{\partial y}\right)-\frac{\partial P}{\partial x} \\
\frac{\partial(\rho u v)}{\partial x}+\frac{\partial(\rho v v)}{\partial y}=\frac{\partial}{\partial x}\left(\mu \frac{\partial v}{\partial x}\right)+\frac{\partial}{\partial y}\left(\mu \frac{\partial v}{\partial y}\right)-\frac{\partial P}{\partial y} \\
\frac{\partial(\rho u)}{\partial x}+\frac{\partial(\rho v)}{\partial y}=0
\end{array}\right.
$$

where $u$ and $v$-horizontal and vertical components of the speed vector.

Boundary conditions for the given system solution are as follows:

1. Conditions at the input to the duct : $x=0,0<y<H: u=$ const, $v=0$.

2. Conditions on the duct walls: $y=0, y=H, 0 \leq x \leq L: u=v=0$.

3. Conditions at the duct output: $x=0,0<x<H: \frac{\partial u}{\partial x}=\frac{\partial v}{\partial x}=0$.

In many cases connected with mathematical simulation of the liquid flow there are regions with irregular geometry (the moist steam flow in APS separators). To solve such tasks either orthogonal coordinate systems (cylindrical, spherical, etc.) or regular mesh with blocked regions in the Cartesian system are used. To solve the tasks with complex geometry the mesh with blocked regions is used in the work [2].

\section{Results}

The results of the straight duct with the size of $1 \times 0.1 \mathrm{~m}$, with the Reynolds number at the duct output equal to $\operatorname{Re}_{H}=150$, which corresponds to the speed at the duct input of $1 \mathrm{~m} / \mathrm{s}$, and the mesh with the dimension of $100 \times 100$ is used, are shown as an example. As the duct is symmetrical, the graphical results of calculations are presented in figure 1 for the half of the duct.

In figure 1 the sections of the speed horizontal component at different distances from the duct input $(2 x / H)$ are shown.

The model verification was carried out by comparing the obtained results with calculation and experimental [4] works of other authors. The comparison showed high assurance of the results obtained by the model.

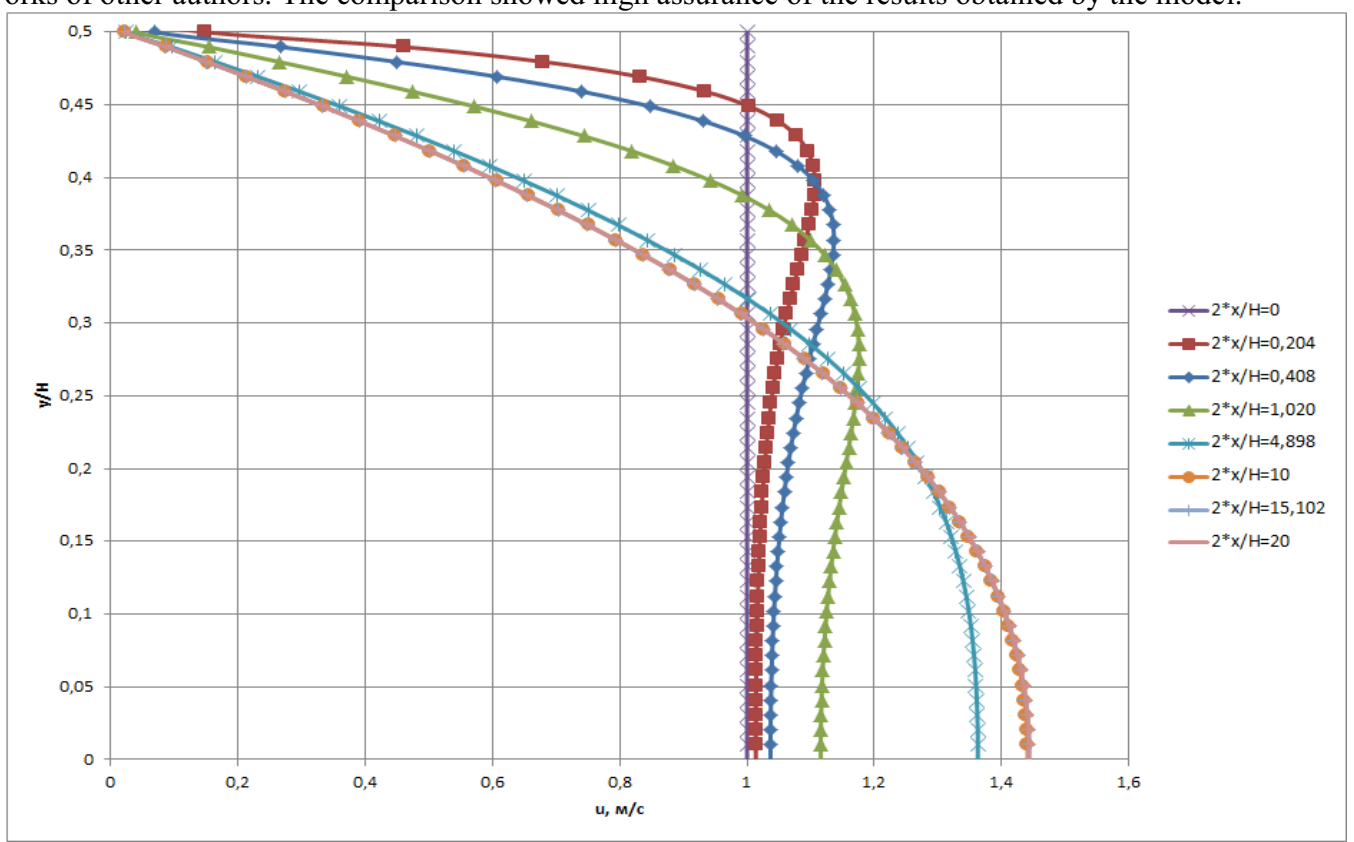

Figure 1. Sections of $u$-speed component in various cross sections of the 2-dimensional duct 
The calculation results for the variable cross-section 2-dimensional duct are presented on the example of the duct the walls of which obey the equations (figure 2):

$$
\begin{aligned}
& f_{1}(x)=0.18 x^{2}, \\
& f_{2}(x)=0.1 x^{2}+0.15,
\end{aligned}
$$

at $x=0 . .1 \mathrm{~m}$.

The duct is placed into the mesh with the size of $L \times H=1 \times 0,3 \mathrm{~m}$ and the number of units $N_{X} \times N_{Y}=150 \times 100$. The input speed is $u=1 \mathrm{~m} / \mathrm{s}$, thus the Reynolds number at the duct input is $\operatorname{Re}_{H}=150$.

With distance from the duct input the speed vector section develops becomes parabolic (figure 2). At the same time, when the duct turns, there appears the vertical speed component and, correspondingly, the speed vector deviates from the horizontal.

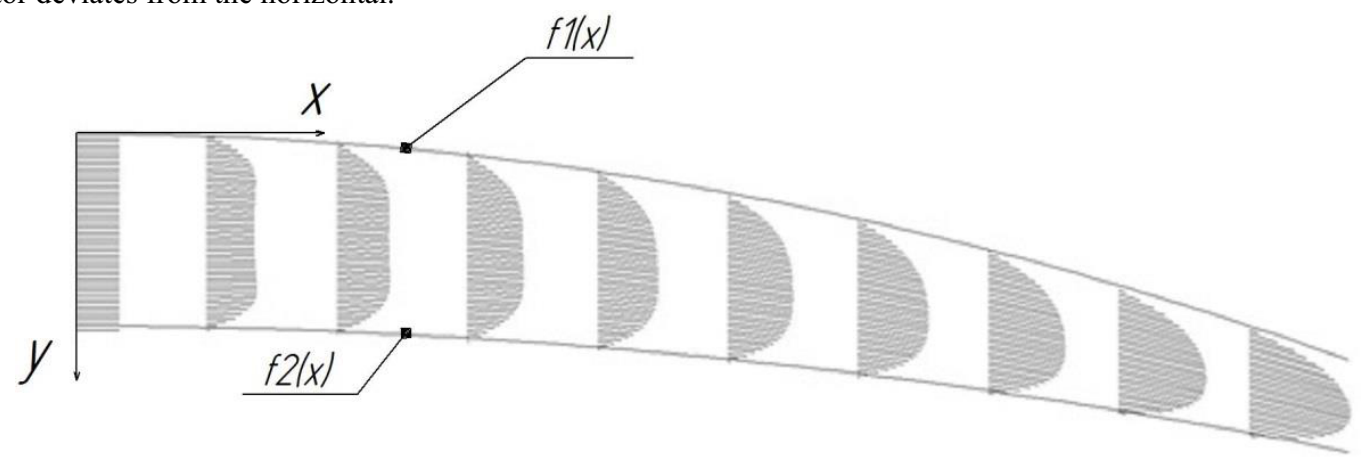

Figure 2. Speed field in the variable cross-section duct

When the speeds field at the gas motion in the duct is determined, it is possible to calculate particles motion in the flow. It is considered in the work that when the particle moves, it is not deformed and influenced only by air-resistance force [3]. Initial position of the particle and its speed are set to calculate the trajectory.

Let us consider the motion of a spherical particle in the gas flow $\left(\operatorname{Re}_{H}=150\right)$, which moves along the duct, the walls of which are set by the following equations:

$$
\begin{aligned}
& f_{1}(x)=3.5 x^{2}, \\
& f_{2}(x)=5 x^{2}-0.4 x+0.06, \\
& \text { at } x=0 . .0,13 \mathrm{~m} .
\end{aligned}
$$

It is supposed that the particle at the duct input ( $x_{0}=0, y_{0}=$ const - initial coordinates) is stationary.

In figure 3 calculated motion trajectories of the drops with the diameters of $100 \mathrm{mcm}$ and $10 \mathrm{mcm}$ for two different initial coordinates $y_{0}=22 \mathrm{~mm} ; 40 \mathrm{~mm}$ are presented. It can be noticed that the particles with the smaller diameter (and smaller mass) pass through the duct easily. The particles with the bigger diameter collide with the firm boundary of the duct. The difference of the motion trajectories is determined mainly by the particles mass. Therefore, a lighter particle accelerates to the speed of carrying media faster and moves along the current line without any contact with the duct walls. 


\section{MATEC Web of Conferences}

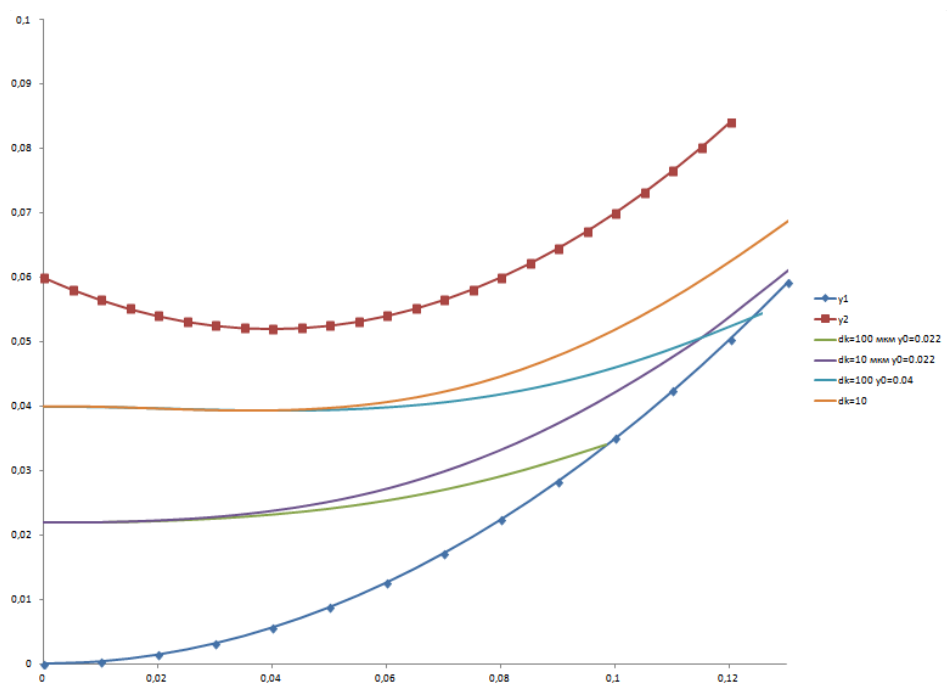

Figure 3. Particles motion trajectories

\section{Conclusion}

The developed mathematical model allows determining the fields of speeds and pressure, when viscous incompressible fluid flows in 2-dimensional variable cross-section ducts at laminar flow mode.

The dynamics of the condensed moisture is determined by the differential equation of the motion amount for the particle. Solving the equation the trajectories of the moisture drops motion and its speed in continuous medium flow.

The work is completed using the funds of the Competitiveness Enhancement Program of TPU (Project_Leading Research University_Institute of Power Engineering_138_2014).

\section{References}

1. Margulova, T. Kh. Atomic power stations: textbook $-5^{\text {th }}$ edition, revised and enlarged Moscow: IzdAT, 1994. - 296 pp.

2. Versteeg H.G., Malalasekera W. //An introduction to computational fluid dynamics// Pearson Education Limited, 2007. - 514 pp.

3. Deich, M. E. Gas dynamics of two-phase media//M.E. Deich, G.A. Philippov. $-2^{\text {nd }}$ edition, revised and enlarged - Moscow: Energoizdat, 1981. - 471 pp.

4. Vinogradova A.I., Zubkov V.G. Gas dynamic processes in heat and power plants on the basis of control volume approach//Mathematical simulation, 6, 3 (2002).

5. Patankar S. V. Numerical Heat Transfer and Fluid Flow. — Taylor \& Francis, 1980 